\title{
An Anomaly Associated with 4-Dimensional Quantum Gravity
}

\author{
Thomas Branson \\ Department of Mathematics, University of Iowa, Iowa City, IA 52242, USA. \\ (E-mail: branson@math.uiowa.edu)
}

Received: 8 June 1995

\begin{abstract}
We compute the functional determinant quotient $\left(\operatorname{det} P_{h}\right) /\left(\operatorname{det} P_{g}\right)$ for the Paneitz operator $P$ in conformally related Riemannian metrics $g, h$, and discuss related positivity questions.
\end{abstract}

\section{Introduction}

In 1983, Paneitz introduced a fourth-order differential operator invariant $P$ of conformal manifolds, which has in many aspects proved to be the analogue of the two-dimensional scalar Laplacian in four-dimensional conformal theory [P]. The original application of this operator was to conformally invariant gauge-fixing for the Maxwell equations, but it has played important roles in the later studies [B1-3, BCY, BØ3, CY, ES1-2]. In [C, IV.4. $\gamma]$, the functional determinant of $P$ is found to define an anomaly associated to a quantum gravitational action defined using the Wodzicki residue [Wo].

In [BØ3], it was shown that determinant quotients for a large class of operators $\mathscr{D}$ which includes $P$ are explicitly computable in dimension 4 . The quotients in question have the form $\left(\operatorname{det} A_{\omega}\right) /\left(\operatorname{det} A_{0}\right)$, where $A \in \mathscr{D}, A_{0}$ is the operator $A$ evaluated in some background metric $g_{0}$, and $A_{\omega}$ is the same operator evaluated in a conformally related metric $g_{\omega}=e^{2 \omega} g_{0}$, where $\omega$ is a $C^{\infty}$ function. The underlying space is a 4-dimensional smooth manifold $M$ equipped with a conformal structure compatible with $g_{0}$. By Schoen's solution of the Yamabe problem [S], we may assume $g_{0}$ has constant scalar curvature. The class $\mathscr{D}$ consists of formally self-adjoint operators with positive definite leading symbol, which are positive integral powers of conformally covariant differential operators. These operators can act on tensor-spinors (the square of the Dirac operator is an example), but in this paper we shall only look at scalar operators. Of course the "operators" we speak of are really functors which canonically assign an operator to each manifold of this type; an example of such a functor is provided by the Yamabe operator (conformal Laplacian). A conceptual point which has been crucial in the later works [B3, BCY, CY] (see also [Be]) is the decision in [BØ3] to use a basis for the invariants appearing in the operator 
asymptotics which is suitably adapted to $P$ (whether or not the operator $A$ whose determinant is being studied is $P$ ).

The purpose of this paper is to compute the determinant quotient for the Paneitz operator. To do this using the methods of [BØ3], one needs a formula for a certain local invariant, specifically, a term from the small- $t$ asymptotic expansion of $\operatorname{Tr}_{L^{2}}\left(f e^{-t P}\right)$ for an arbitrary smooth function $f$. The best results on asymptotics of differential operators with leading symbol $|\xi|^{4}$, due to Gilkey [G], only provide integrated asymptotics (from the expansion of $\operatorname{Tr}_{L^{2}} e^{-t P}$ ) at the required order. However, given conformal covariance, we can use a principle introduced in [BØ1] to reconstruct the local information from the integrated information in dimensions $m \neq 4$, and observe its dependence on the dimension. By the recursive construction of the asymptotics, this should be rational in $m$ (after division by a certain meromorphic factor determined by the leading symbol). Thus the formula for $m \neq 4$ leads to one for $m=4$ by meromorphic continuation, with $m=\infty$ as the limit point.

\section{The Determinant of the Paneitz Operator}

Let $(M, g)$ be a Riemannian manifold of dimension $m \geqq 3$, with Riemannian curvature $R$, Ricci curvature $\rho$, and scalar curvature $\tau$. Our convention on placement of indices has $R_{212}^{1}>0$ on standard spheres. Let $d$ be the exterior derivative and $\delta$ its formal adjoint; the Laplacian on scalar functions is then $\Delta=\delta d$. Let

$$
\begin{aligned}
J & =\tau /(2(m-1)), \\
V & =(\rho-J g) /(m-2), \\
T & =(m-2) J-4 V \cdot, \\
Q & =m J^{2} / 2-2|V|^{2}+\Delta J .
\end{aligned}
$$

Here $V \cdot$ is the natural action of a two-tensor on one-tensors, i.e $(V \cdot \varphi)_{j}=V^{k}{ }_{j} \varphi_{k}$, and $|V|^{2}=V^{j k} V_{j k}$. The Weyl conformal curvature tensor $C$ is defined by

$$
R^{i}{ }_{j k l}=C^{i}{ }_{j k l}-V_{j k} \delta_{l}^{i}+V_{j l} \delta_{k}^{i}-V^{i}{ }_{l} g_{j k}+V^{i}{ }_{k} g_{j l} .
$$

The Paneitz operator is

$$
P=\Delta^{2}+\delta T d+\frac{m-4}{2} Q
$$

$P$ is conformally covariant in the sense that

$$
g_{\omega}=e^{2 \omega} g_{0}, \quad \omega \in C^{\infty}(M) \Rightarrow P_{\omega}=e^{-(m+4) \omega / 2} P_{0}\left[e^{(m-4) \omega / 2}\right] .
$$

Here and below, for any $F \in C^{\infty}(M),[F]$ is the multiplication operator $f \mapsto F f$.

Note the analogy with the conformal Laplacian

$$
Y=\Delta+\frac{m-2}{2} J
$$

which satisfies the conformal covariance relation

$$
Y_{\omega}=e^{-(m+2) \omega / 2} Y_{0}\left[e^{(m-2) \omega / 2}\right] .
$$

The quantity $Q$ is not really visible when we view $P$ only in dimension 4; similarly the Gauss curvature $J$ does not show up in $Y$ for $m=2$. But $Q$ in dimension 4 , like $J$ in dimension 2, gives rise to a curvature prescription problem which puts us in 
contact with exponential class inequalities of Moser-Trudinger type [B3]. (See (13) and Sect. 3 below.) As shown in [GJMS], $Y$ and $P$ generalize to a sequence $P_{n}$ of conformally covariant differential operators of even order $n$, defined whenever $m$ is odd or $m \geqq n$.

Suppose $A$ is a formally self-adjoint differential operator with positive definite leading symbol. Then the order of $A$ is necessarily an even number $2 l$. Suppose $l>0$ and let $f$ be a smooth function. Recall the heat expansion

$$
\operatorname{Tr}_{L^{2}}\left(f e^{-t A}\right) \sim \sum_{j=0}^{\infty} t^{(j-m) / 2 l} \int f U_{j}[A] d v, \quad t \downarrow 0,
$$

where $d v$ is the Riemannian measure, and the $U_{j}[A]$ are smooth invariants of the total symbol of $A$. (More specifically, $U_{j}[A]$ is polynomial in the total symbol, with coefficients that are smooth in the leading symbol.) With this labelling of the heat invariants, the $U_{\text {odd }}$ vanish, and $U_{m}$ is the coefficient of $t^{0}$ (and thus is the invariant at the index level).

Lemma 1 [BØ1]. If $A$ is conformally covariant in the sense $g_{\omega}=e^{2 \omega} g_{0} \Rightarrow \bar{A}_{\omega}=$ $e^{-b \omega} A_{0}\left[e^{a \omega}\right]$ for any smooth $\omega$, or if $A$ is a positive integral power of a conformal covariant, then for the variation $g_{\varepsilon \omega}=e^{2 \varepsilon \omega} g_{0}$,

$$
\left.\frac{d}{d \varepsilon}\right|_{\varepsilon=0} \int\left(U_{j}[A] d v\right)_{\varepsilon \omega}=(m-j) \int \omega\left(U_{j}[A] d v\right)_{0} .
$$

In particular, $\int U_{m}[A] d v$ is a conformal invariant.

By Weyl's invariant theory [We], local scalar $\mathrm{O}(m)$ invariants of the homogeneity of $U_{4}[A]$ are linear combinations of the four invariants $|R|^{2},|\rho|^{2}, \tau^{2}$, and $\Delta \tau$. After a change of basis, these are linear combinations of $|C|^{2}, Q, J^{2}$, and $\triangle J$. Using $\left.(d / d \varepsilon)\right|_{\varepsilon=0}(d v)_{\varepsilon \omega}=m \omega(d v)_{0}$, the conformal invariance of $C$, and the conformal covariance relation (3) for $P$, we get

$$
\begin{gathered}
\left.\frac{d}{d \varepsilon}\right|_{\varepsilon=0} \int\left(|C|^{2} d v\right)_{\varepsilon \omega}=(m-4) \int \omega\left(|C|^{2} d v\right)_{0}, \\
\left.\frac{d}{d \varepsilon}\right|_{\varepsilon=0} \int(Q d v)_{\varepsilon \omega}=(m-4) \int \omega(Q d v)_{0} .
\end{gathered}
$$

Actually, (5) does not follow immediately from (3) when $m=4$, but one can analytically continue the relation, which is rational in $m$, to $m=4$.

$\Delta J$ is an exact divergence, so its integral is universally 0 . The conformal covariance relation for $Y$ shows that $\left.(d / d \varepsilon)\right|_{\varepsilon=0} J_{\varepsilon \omega}+2 \omega J_{0}=\Delta \omega$; thus

$$
\begin{aligned}
\left.\frac{d}{d \varepsilon}\right|_{\varepsilon=0} \int\left(J^{2} d v\right)_{\varepsilon \omega} & =(m-4) \int \omega\left(J^{2} d v\right)_{0}+2 \int\left(\Delta_{0} \omega\right)(J d v)_{0} \\
& =(m-4) \int \omega\left(J^{2} d v\right)_{0}+2 \int \omega((\Delta J) d v)_{0} .
\end{aligned}
$$

Thus if

$$
U_{4}[A]=\beta_{0} J^{2}+\beta_{1}|C|^{2}+\beta_{2} Q+\beta_{3} \Delta J, \quad \beta_{j}=\beta_{j}[m, A],
$$

then

$$
\begin{aligned}
\left.\frac{d}{d \varepsilon}\right|_{\varepsilon=0} \int\left(U_{4}[A] d v\right)_{\varepsilon \omega} & =(m-4) \int \omega\left(U_{4}[A] d v\right)_{0} \\
& +\left(2 \beta_{0}-(m-4) \beta_{3}\right) \int \omega((\Delta J) d v)_{0} .
\end{aligned}
$$


If $A$ is conformally covariant or a positive integral power of such, the fact that (7) holds for all $(M, g, \omega)$, together with Lemma 1, yields

$$
2 \beta_{0}[m, A]=(m-4) \beta_{3}[m, A] .
$$

In particular, under this conformal assumption on $A$, we have $\beta_{0}[4, A]=0$.

Lemma 2. Let

Then

$$
u(m):=720\left(m^{2}-4\right)(4 \pi)^{m / 2} \frac{\Gamma\left(\frac{m-4}{2}\right)}{\Gamma\left(\frac{m-4}{4}\right)}
$$

$$
\begin{aligned}
U_{4}[P]= & u(m)^{-1}\left\{-(m-8)(m+4)(m+2)(m-12)\left[\Delta J+\frac{1}{2}(m-4) J^{2}\right]\right. \\
& \left.+(m-8)\left(m^{3}-52 m-24\right) Q+2\left(m^{2}-4\right)|C|^{2}\right\} .
\end{aligned}
$$

Proof. To restate, the assertion is that

$$
\begin{aligned}
& u(m) \beta_{0}[m, P]=-\frac{1}{2}(m-4)(m-8)(m+4)(m+2)(m-12), \\
& u(m) \beta_{1}[m, P]=2\left(m^{2}-4\right), \\
& u(m) \beta_{2}[m, P]=(m-8)\left(m^{3}-52 m-24\right), \\
& u(m) \beta_{3}[m, P]=-(m-8)(m+4)(m+2)(m-12) .
\end{aligned}
$$

Gilkey [G] shows that if $A$ is a scalar differential operator of the form

$$
A f=\Delta^{2} f+\langle p, \nabla \nabla f\rangle+\langle q, \nabla f\rangle+r f,
$$

where $p$ is a smooth symmetric section of $T M \otimes T M, q$ is a smooth vector field, $r$ is a smooth function, and $\langle\cdot, \cdot\rangle$ is the natural dual pairing, then

$$
\begin{aligned}
\int U_{4}[A] d v= & u(m)^{-1} \int\left\{\left(m^{2}-4\right)\left[5 \tau^{2}-2|\rho|^{2}+2|R|^{2}\right]\right. \\
& +30(m+2)[\tau\langle g, p\rangle-2\langle\rho, p\rangle]+45\langle g, p\rangle^{2} \\
& \left.+90|p|^{2}-360(m+2) r\right\} .
\end{aligned}
$$

Note that the coefficient $q$ from (10) does not appear in the formula for $\int U_{4}$. As noted in $[\mathrm{G}]$, some invariant theory shows that it can only appear in pure divergence terms in $U_{4}$.

Now let $A=P$. By (1) and the Bianchi identity $\nabla^{j} V_{j k}=\nabla_{k} J$, the coefficients in (10) are given by

$$
p_{b}=4 V-(m-2) J g, \quad q_{b}=(6-m) \nabla J, \quad r=\frac{m-4}{2} Q,
$$

where $b$ denotes the metric identification of contravariant with covariant tensor bundles. Since $V_{j}^{j}=J$ and $C$ is orthogonal to the rest of the right-hand side in (2),

$$
\begin{aligned}
\langle g, p\rangle & =-\left(m^{2}-2 m-4\right) J, \\
|p|^{2} & =16|V|^{2}+(m-4)\left(m^{2}-4\right) J^{2}, \\
\tau\langle g, p\rangle & =-2(m-1)\left(m^{2}-2 m-4\right) J^{2},
\end{aligned}
$$




$$
\begin{gathered}
\langle\rho, p\rangle=4(m-2)|V|^{2}-2 m(m-3) J^{2}, \\
|\rho|^{2}=(m-2)^{2}|V|^{2}+(3 m-4) J^{2}, \\
|R|^{2}=|C|^{2}+4(m-2)|V|^{2}+4 J^{2} .
\end{gathered}
$$

Equation (11) and these formulas show that modulo $\mathscr{R}(\delta)$,

$$
\begin{aligned}
u(m) U_{4}[P] \simeq & (m-8)\left[-2\left(m^{3}-52 m-24\right)|V|^{2}\right. \\
& \left.+\left(5 m^{3}-6 m^{2}-92 m-192\right) J^{2}\right]+2\left(m^{2}-4\right)|C|^{2} \\
\simeq & (m-8)\left[\left(m^{3}-52 m-24\right) Q-\frac{1}{2}(m-4)(m+4)(m+2)\right. \\
& \left.\times(m-12) J^{2}\right]+2\left(m^{2}-4\right)|C|^{2} .
\end{aligned}
$$

This gives the desired formulas (9) for $\beta_{j}[m, P]$, except for $\beta_{3}$. For $m \neq 4$, the formula for $\beta_{3}$ follows from that for $\beta_{0}$, together with (8). We can continue the formula for $\beta_{3}$ to $m=4$ by examining the recursion that generates $U_{4}$ from the total symbol: for the leading symbol $|\xi|^{4}$, the quantity $u(m) U_{4}[A]$ is polynomial in the coefficients of $A$. Since the coefficients of $P$ and $V$ are rational in $m$, the $\beta_{j}[m, P]$ are also.

Note that $u(m)$ is regular at $m=4$, with

so that

$$
u(4)=720 \cdot 12 \cdot(4 \pi)^{2} \cdot \frac{1}{2}=69120 \pi^{2},
$$

$$
720 \pi^{2}\left(\beta_{1}[4, P], \beta_{2}[4, P], \beta_{3}[4, P]\right)=\left(\frac{1}{4}, 7,-16\right) .
$$

As noted after $(8), \beta_{0}[4, P]=0$ is a consequence of conformal covariance.

Back in the general setting, if $A$ is positive definite, the functional determinant is defined by $\operatorname{det} A=e^{-\zeta_{A}^{\prime}(0)}$, where $\zeta_{A}(s)$ is the analytic continuation of $\operatorname{Tr}_{L^{2}} A^{-s}$. Under our analytic assumptions of formal self-adjointness and leading symbol $|\xi|^{4}$, we do not quite have positive definiteness; there may be finitely many negative and 0 eigenvalues. By [BØ2], if $A$ is conformally covariant or a power of such, the number of negative eigenvalues (counted with multiplicity) and the multiplicity of 0 as an eigenvalue are conformal invariants. We may define $\operatorname{det} A$ by

$$
-\log |\operatorname{det} A|=\zeta_{A}^{\prime}(0), \quad \operatorname{sgn} \operatorname{det} A=\#\left\{\lambda_{\mathrm{j}}=0\right\},
$$

where $\lambda_{j}$ are the eigenvalues of $A$, labelled with multiplicity, and $\zeta_{A}(s)$ is the analytic continuation of $\sum_{\lambda_{j} \neq 0}\left|\lambda_{j}\right|^{-s}$. We then get:

Lemma 3 [BØ2, PR]. If $A$ satisfies the analytic assumptions above and is conformally covariant or a power of such, then

$$
\left.\frac{d}{d \varepsilon}\right|_{\varepsilon=0} \zeta_{A_{\varepsilon \omega}}^{\prime}(0)=2 l \int \omega\left\{U_{m}[A]-\sum_{\lambda_{j}=0}\left|\varphi_{j}\right|^{2}\right\} d v,
$$

where $\left\{\left(\lambda_{j}, \varphi_{j}\right)\right\}$ is an orthonormal spectral resolution of $A$.

This variational property uniquely determines

$$
\zeta_{A_{\omega}}^{\prime}(0)-\zeta_{A_{0}}^{\prime}(0)=-\log \frac{\operatorname{det} A_{\omega}}{\operatorname{det} A_{0}}
$$


for all smooth $\omega$, since one can compute $\zeta_{A_{\varepsilon \omega}}^{\prime}(0)$ as the solution of an ordinary differential equation in the parameter $\varepsilon$. Thus one knows $\operatorname{det} A$ as a functional on the conformal class $\left\{e^{2 \omega} g_{0} \mid \omega \in C^{\infty}(M)\right\}$, up to a constant factor. The determinant supplies the missing conformal primitive (integral) for $U_{m}[A]$ not provided by Lemma 1.

To solve these ordinary differential equations, first write $P=\tilde{P}+(m-4) Q / 2$. Then $\tilde{P}$ annihilates constants, and equals $P$ in dimension 4 . Applying the conformal covariance relation (3) to the function 1 , we get

$$
\begin{aligned}
\frac{m-4}{2} Q_{\omega} & =e^{-(m+4) \omega / 2} \tilde{P}_{0} e^{(m-4) \omega / 2}+\frac{m-4}{2} e^{-4 \omega} Q_{0} \\
& =e^{-(m+4) \omega / 2} \tilde{P}_{0}\left(e^{(m-4) \omega / 2}-1\right)+\frac{m-4}{2} e^{-4 \omega} Q_{0} .
\end{aligned}
$$

Analytically continuing to dimension 4 , we get

$$
P_{0} \omega+Q_{0}=e^{4 \omega} Q_{\omega}, \quad m=4 .
$$

This is the Paneitz curvature prescription equation, in full analogy with the Gauss curvature prescription equation $\Delta_{0} \omega+J_{0}=e^{2 \omega} J_{\omega}$ in dimension 2 . This shows that in dimension 4 , the functional

$$
\mathscr{P}(\omega):=\frac{1}{2} \int \omega\left(P_{0} \omega\right)(d v)_{0}+\int \omega(Q d v)_{0}
$$

solves the relevant ordinary differential equation, namely

$$
\left.\frac{d}{d \varepsilon}\right|_{\varepsilon=\varepsilon_{0}} \mathscr{P}(\varepsilon \omega)=\int \omega(Q d v)_{\varepsilon_{0} \omega} .
$$

Since $\left(|C|^{2} d v\right)_{\omega}$ is independent of $\omega$ in dimension 4 ,

$$
\left.\frac{d}{d \varepsilon}\right|_{\varepsilon=\varepsilon_{0}} \int \varepsilon \omega\left(|C|^{2} d v\right)_{\varepsilon \omega}=\int \omega\left(|C|^{2} d v\right)_{\varepsilon_{0} \omega} .
$$

By (6) with $g_{\varepsilon_{0} \omega}$ in place of $g_{0}$,

$$
\left.\frac{d}{d \varepsilon}\right|_{\varepsilon=\varepsilon_{0}} \int\left(J^{2} d v\right)_{\varepsilon \omega}=2 \int \omega((\Delta J) d v)_{\varepsilon_{0} \omega}
$$

for $m=4$.

In dimension 4 , we always have $1 \in \mathscr{N}(P)$. We make the simplifying assumption that this is the whole null space; that is, we work on a manifold $\left(M, g_{0}\right)$ satisfying the conformally invariant condition $\mathscr{N}(P)=\mathbb{R} 1$. If $v=\int d v$ is the total volume, then the constant function $v^{-1 / 2}$ is an orthonormal basis for $\mathscr{N}(P)$. The computation

$$
\left.\frac{d}{d \varepsilon}\right|_{\varepsilon=\varepsilon_{0}} \log v_{\varepsilon \omega}=\int 4 \omega\left(v^{-1} d v\right)_{\varepsilon_{0} \omega}
$$

thus solves the ordinary differential equation corresponding to the last term in (12). With Lemmas 2 and 3, these computations give: 
Theorem 4. On a compact conformal 4-manifold with $\mathscr{N}(P)=\mathbb{R} 1$,

$$
\begin{aligned}
-\log \frac{\operatorname{det} P_{\omega}}{\operatorname{det} P_{0}}= & \frac{1}{720 \pi^{2}}\left[28\left\{\frac{1}{2} \int \omega\left(P_{0} \omega\right)(d v)_{0}+\int \omega(Q d v)_{0}\right\}\right. \\
& \left.-32 \int\left\{\left(J^{2} d v\right)_{\omega}-\left(J^{2} d v\right)_{0}\right\}+\int \omega\left(|C|^{2} d v\right)_{0}\right]-\log \frac{v_{\omega}}{v_{0}} .
\end{aligned}
$$

\section{The Extremal Problem}

Suppose we would like to find the extremals of the functional determinant within a conformal class. The job as stated cannot be done, since the determinant is not invariant under uniform scaling of the metric. If $\alpha$ is a constant, $\zeta_{A_{\alpha}}(s)=$ $\zeta_{e^{-2 l \alpha} A_{0}}(s)=e^{2 l \alpha s} \zeta_{A_{0}}(s)$; thus $\zeta_{A_{\alpha}}^{\prime}(0)=\zeta_{A_{0}}^{\prime}(0)+2 l \alpha$. This problem can be cured by restricting to metrics of a certain fixed volume, or (what amounts to the same thing), shifting attention to the scale-invariant zeta function

$$
\tilde{\zeta}_{A_{\omega}}(s):=\left(\frac{v_{\omega}}{v_{0}}\right)^{-2 l s / m} \zeta_{A_{\omega}}(s),
$$

for which

$$
\tilde{\zeta}_{A_{\omega}}^{\prime}(0)=\zeta_{A_{\omega}}^{\prime}(0)-\frac{2 l}{m} \zeta_{A_{\omega}}(0) \log \left(\frac{v_{\omega}}{v_{0}}\right) .
$$

If $A$ is conformally covariant, $\zeta_{A}(0)$ is a conformal invariant; in fact $\zeta_{A}(0)=$ $-\operatorname{dim} \mathscr{N}(A)+\int U_{m}[A] d v$. (Recall Lemma 1 and the discussion above.) With Theorem 4, this shows that on a compact conformal 4-manifold with $\mathscr{N}(P)=\mathbb{R} 1$,

$$
\begin{aligned}
-\log \frac{\operatorname{det} P_{\omega}}{\operatorname{det} P_{0}}= & \frac{1}{720 \pi^{2}}\left[28\left\{\frac{1}{2} \int \omega\left(P_{0} \omega\right)(d v)_{0}+\int \omega(Q d v)_{0}\right\}\right. \\
& \left.-32 \int\left\{\left(J^{2} d v\right)_{\omega}-\left(J^{2} d v\right)_{0}\right\}+\int \omega\left(|C|^{2} d v\right)_{0}\right] \\
& -\left(\int\left(U_{4}[P] d v\right)_{0}\right) \log \frac{v_{\omega}}{v_{0}},
\end{aligned}
$$

where dêt is defined to correspond to $\tilde{\zeta}$.

If $g_{0}$ is locally symmetric (i.e. $\left.(\nabla R)_{0}=0\right)$, then all local scalar invariants are constant when evaluated at $g_{0}$. The last equation can then be rewritten

$$
\begin{aligned}
-\log \frac{\operatorname{det} P_{\omega}}{\operatorname{det} P_{0}}= & \frac{v_{0}}{720 \pi^{2}}\left[7\left\{2 \int \omega\left(P_{0} \omega\right)(d v)_{0}-Q_{0} \log \int e^{4(\omega-\bar{\omega})}(d v)_{0}\right\}\right. \\
& -32 \chi\left\{\left(J^{2} d v\right)_{\omega}-\left(J^{2} d v\right)_{0}\right\}-\frac{1}{4}\left(|C|^{2}\right)_{0} \log \left\{e^{4(\omega-\bar{\omega})}(d v)_{0}\right],(1
\end{aligned}
$$

where $\bar{\omega}$ is the $(d v)_{0}$ average of $\omega$, and $\mathcal{X}:=v_{0}^{-1} \int$.

From the point of view of the extremal problem, this functional would seem to be more subtle than the corresponding functionals for the conformal Laplacian $Y$ and the square $\varnothing^{2}$ of the Dirac operator studied in [BCY, BØ3]. This is because the signs of the coefficients of the constituent functionals

$$
\begin{aligned}
& \mathscr{F}_{1}(\omega):=\frac{1}{3} \oint \omega\left(P_{0} \omega\right)(d v)_{0}-\frac{1}{6} Q_{0} \log \int e^{4(\omega-\bar{\omega})}(d v)_{0}, \\
& \mathscr{F}_{2}(\omega):=\mathcal{X}\left\{\left(J^{2} d v\right)_{\omega}-\left(J^{2} d v\right)_{0}\right\}
\end{aligned}
$$


differ. On $M=S^{4}$, with $g_{0}$ the standard round metric (where $Q_{0}=6, J_{0}=2$, and $P_{0}=\Delta_{0}\left(\Delta_{0}+2\right)$ ), both $\mathscr{F}_{1}$ and $\mathscr{F}_{2}$ are nonnegative by virtue of two sharp inequalities with the same extremal functions:

Lemma 5 [Be, BCY, CL]. On $S^{4}$, if $g_{0}$ is the round metric, then $\mathscr{F}_{i}(\omega) \geqq 0, i=1,2$, with equality if and only if $g_{\omega}=\left(h^{-1}\right)^{*} g_{0}$, where $h$ is a conformal diffeomorphism.

Thus there is a finite-parameter family of extremals in each case, as the conformal group of $\left(S^{4}, g_{0}\right)$ is isomorphic to $\mathrm{SO}(5,1)$. If $g_{\omega}=\left(h^{-1}\right)^{*} g_{0}$ for a conformal diffeomorphism $h$, we shall call $\omega$ a conformal factor. The scale-adjusted determinant quotients for $Y$ and $\not^{2}$ on $S^{4}$ have the form $c_{1} \mathscr{F}_{1}(\omega)+c_{2} \mathscr{F}_{2}(\omega)$ for $\operatorname{sgn} c_{1}=\operatorname{sgn} c_{2}$; thus the sharp inequalities expressed in Lemma 5 are immediately decisive in these cases.

As noted in [B3], the $\mathscr{F}_{2}$ inequality is a covariant form of the norm calculation for the embedding $L_{1}^{2} \hookrightarrow L^{4}$, while the $\mathscr{F}_{1}$ inequality similarly governs the embedding $L_{2}^{2} \hookrightarrow e^{L}$ (where the $L_{r}^{2}$ are Sobolev classes, and $e^{L}$ is meant in the sense of Orlicz classes). The $L_{2}^{2} \hookrightarrow e^{L}$ embedding may be viewed as an endpoint derivative of the borderline Sobolev embeddings $L_{r}^{2} \hookrightarrow L^{4 /(2-r)}$ as $r \uparrow 2$ [Be]. Each such embedding in turn has an interpretation as a comparison of two invariant norms on a complementary series representations of $\mathrm{SO}(5,1)$; the process $r \uparrow 2$ takes us to the endpoint, or first point of reducibility, for the complementary series [B3]. In fact, the fact that $P$ annihilates constants is an expression of this reducibility.

By virtue of the sign agreement mentioned above, it was possible to show in [BCY] that the suitably scale-adjusted determinant of $Y$ (respectively $\varnothing^{2}$ ) is minimized (respectively maximized) exactly at the round metric and its $\operatorname{SO}(5,1)$ transforms. For the scale-adjusted determinant of $P$, one must study the relative size of $\mathscr{F}_{1}(\omega)$ and $\mathscr{F}_{2}(\omega)$; i.e., of the gaps between the two sides in two sharp inequalities.

Conjecture 6. On $S^{4}$, in the conformal class of the round metric $g_{0}$, the functional $\operatorname{det} P_{\omega}$ is minimized exactly when $\omega$ is a conformal factor.

As evidence for this, we have:

Lemma 7. On $S^{4}$, the second variation of $\left(\mathscr{F}_{2}+a \mathscr{F}_{1}\right)(\omega)$ at $\omega=0$ is positive semidefinite if $a \geqq-15 / 8$, indefinite if $-3<a<-15 / 8$, and negative semidefinite if $a \leqq-3$.

Proof. Without loss of generality, we may assume $\bar{\omega}=0$; that is, $\omega \perp 1$ in $L^{2}$. With all operators and measures computed in the round metric,

$$
\begin{aligned}
& \log \int e^{4 \varepsilon \omega}=8 \varepsilon^{2} \int \omega^{2}+O\left(\varepsilon^{3}\right) \text {, } \\
& \mathscr{F}_{1}(\varepsilon \omega)=\frac{1}{3} \varepsilon^{2}\left\{\omega\{\Delta(\Delta+2)-24\} \omega+O\left(\varepsilon^{3}\right)=\frac{1}{3} \varepsilon^{2} \int \omega(\Delta+6)(\Delta-4) \omega+O\left(\varepsilon^{3}\right),\right. \\
& \mathscr{F}_{2}(\varepsilon \omega)=\varepsilon^{2}\left(-4 \chi|d \omega|^{2}+\chi(\Delta \omega)^{2}\right)+O\left(\varepsilon^{3}\right)=\varepsilon^{2} \chi \omega \Delta(\Delta-4) \omega+O\left(\varepsilon^{3}\right) .
\end{aligned}
$$

(One way to get the formula for $\mathscr{F}_{2}(\omega)$ is to apply the conformal covariance relation for $Y$ to the function 1.) Thus

$$
\begin{aligned}
\left(\mathscr{F}_{2}+a \mathscr{F}_{1}\right)(\varepsilon \omega) & =\varepsilon^{2} \int \omega\left\{\left(1+\frac{1}{3} a\right) \Delta+2 a\right\}(\Delta-4) \omega+O\left(\varepsilon^{3}\right) \\
& =\varepsilon^{2}\left\{\omega\left\{\left(1+\frac{1}{3} a\right)(\Delta-10)+10+\frac{16}{3} a\right\}(\Delta-4) \omega+O\left(\varepsilon^{3}\right) .\right.
\end{aligned}
$$


The significance of the operators $\Delta-4$ and $\Delta-10$ is that $0,4,10$ are the bottom eigenvalues of $\Delta$. The lemma follows upon examination of the signs of $1+\frac{1}{3} a$ and $10+\frac{16}{3} a$.

The Laplace eigenfunction corresponding to the 0 eigenvalue is $1 \perp \omega$. The eigenfunctions with eigenvalue 4 are the homogeneous coordinate functions; these are first-order approximations to the conformal factors, which are known to give 0 upon application of either $\mathscr{F}_{i}$. In fact, the conformal factors are the $\log (\cosh t+$ $\left.(\sinh t) x_{5}\right)$ and their images under the rotation group $\mathrm{SO}(5)$; here $t$ runs through $\mathbb{R}$, and $\left(x_{j}\right)$ are the homogeneous coordinate functions. By (14), the determinant functional of interest has $a=-21 / 16$, in the positive semidefinite range.

\section{References}

[Be] Beckner, W.: Sharp Sobolev inequalities on the sphere and the Moser-Trudinger inequality. Ann. Math. 138, 213-242 (1993)

[B1] Branson, T.: Differential operators canonically associated to a conformal structure. Math. Scand. 57, 293-345 (1985)

[B2] Branson, T.: Group representations arising from Lorentz conformal geometry. J. Funct. Anal. 74, 199-291 (1987)

[B3] Branson, T.: Sharp inequalities, the functional determinant, and the complementary series. Trans. Am. Math. Soc. 347, 3671-3742 (1995)

[BCY] Branson, T., Chang, S.-Y.A., Yang, P.: Estimates and extremals for zeta function determinants on four-manifolds. Commun. Math. Phys. 149, 241-262 (1992)

[BØ1] Branson, T., Ørsted, B.: Conformal indices of Riemannian manifolds. Compos. Math. 60, 261-293 (1986)

[BØ2] Branson, T., Ørsted, B.: Conformal geometry and global invariants. Diff. Geom. Appl. 1, 279-308 (1991)

[BØ3] Branson, T., Ørsted, B.: Explicit functional determinants in four dimensions. Proc. Am. Math. Soc. 113, 669-682 (1991)

[CL] Carlen, E., Loss, M.: Competing symmetries, the logarithmic HLS inequality and Onofri's inequality on $S^{n}$. Geom. and Funct. Anal. 2, 90-104 (1992)

[CY] Chang, S.-Y.A., Yang, P.: Extremal metrics of zeta function determinants on 4-manifolds. Ann. Math. (to appear)

[C] Connes, A.: Noncommutative Geometry. San Diego: Academic Press, 1994

[ES1] Eastwood, M., Singer, M.: A conformally invariant Maxwell gauge. Phys. Lett. 107A, 73-74 (1985)

[ES2] Eastwood, M., Singer, M.: The Frölicher spectral sequence on a twistor space. J. Diff. Geom. 38, 653-669 (1993)

[G] Gilkey, P.: The spectral geometry of the higher order Laplacian. Duke Math. J. 47, 511-528 (1980)

[GJMS] Graham, C.R., Jenne, R., Mason, L., Sparling, G.: Conformally invariant powers of the Laplacian, I: Existence. J. London Math. Soc. 46, 557-565 (1992)

[P] Paneitz, S.: A quartic conformally covariant differential operator for arbitrary pseudoRiemannian manifolds. Preprint, 1983

[PR] Parker, T., Rosenberg, S.: Invariants of conformal Laplacians. J. Diff. Geom. 25, 199-222 (1987)

[S] Schoen, R.: Conformal deformation of a Riemannian metric to constant scalar curvature. J. Diff. Geom. 20, 479-495 (1984)

[We] Weyl, H.: The Classical Groups: Their Invariants and Representations. Princeton, NJ: Princeton University Press, 1939

[Wo] Wodzicki, M.: Local invariants of spectral asymmetry. Invent. Math. 75, 143-177 (1984) 
